\title{
Prévalence De La Douleur Neuropathique Chez Des Patients Souffrant De Lomboradiculalgie Commune En Consultation Rhumatologique À Lomé (Togo)
}

\author{
Kodjo Kakpovi, \\ Service de Rhumatologie, CHU Sylvanus Olympio, Lomé-Togo \\ Komi Cyrille Tagbor, \\ Service de Rhumatologie, Hôpital de Bè, Lomé-Togo \\ EyramFianyo, \\ Service de Rhumatologie, CHR Lomé Commune, Lomé-Togo \\ Agbeko Komlan Doleagbenou, \\ Service de neurochirurgie, CHU Sylvanus Olympio, Lomé-Togo \\ Kokou Mensah Guinhouya, \\ Service de neurologie, CHU Sylvanus Olympio, Lomé-Togo \\ Viwalé Etonam Sika Koffi-Tessio,
}

Service de Rhumatologie, CHU Sylvanus Olympio, Lomé-Togo

Prénam Houzou,

Service de Rhumatologie, CHU Kara, Kara-Togo

Essossinam Kpelao,

Service de neurochirurgie, CHU Sylvanus Olympio, Lomé-Togo

Mofou Belo,

Service de neurologie, CHU Sylvanus Olympio, Lomé-Togo

Owonayo Oniankitan,

Service de Rhumatologie, CHR Lomé Commune, Lomé-Togo

Moustafa Mijiyawa,

Service de Rhumatologie, CHU Sylvanus Olympio, Lomé-Togo

doi: 10.19044/esj.2017.v13n24p267 URL:http://dx.doi.org/10.19044/esj.2017.v13n24p267

\begin{abstract}
Objective: To determine the prevalence and factors associated with neuropathic pain in patients with non-specific low back pain. Methods: This was a cross-sectional study conducted from May to July 2016 in the Rheumatology, Neurology and Neurosurgery departments of Lome. The DN4 questionnaire was used for the diagnosis of neuropathic pain in the 200 patients with low back pain included in this study. Results: Of the 200 patients (147 women and 53 men) included in the study, neuropathic pain
\end{abstract}


was present in $92(46 \%)$. The average age of the 92 patients (67 women vs 25 men, $\mathrm{p}=0.04$ ) was $55.5 \pm 12.4$ years (women $55.2 \pm 12.8$ vs. men $54.6 \pm$ $11.4, \mathrm{p}=0.5)$. The characteristics of neuropathic pain mainly found were: burning sensation $(n=67,72.8 \%)$; electrical discharges $(n=64,69.6 \%)$; tingling $(n=90 ; 97.8 \%)$; tickling $(n=57 ; 62 \%)$; numbness $(n=89 ; 96.7 \%)$; hypoesthesia $(\mathrm{n}=52 ; 56.5 \%)$. Factors significantly associated with the presence of neuropathic pain in LBP were age $(p=0.005)$, duration of LBP $(\mathrm{p}=0.04)$, high blood pressure $(\mathrm{p}=0.001)$, radicular pain $(\mathrm{p}=0.00002)$ and the past history of the LBP (0.000000). Conclusion: Neuropathic pain is common in patients with LBP at Lome. The duration of LBP, past history of LBP, previous NSAID use, BMI, pain severity and radicular pain appear to be predictive of the occurrence of these neuropathic pains.

Keywords: Low back pain, neuropathic pain, sub-Saharan Africa

\section{Resume}

Objectif : Déterminer la prévalence et les facteurs associés à la douleur neuropathique (DN) chez des patients ayant une lomboradiculalgie commune (LC).Patients et Méthodes : Il s'est agi d'une étude transversale menée de mai à juillet 2016 dans les services de rhumatologie, de neurologie et de neurochirurgie de Lomé. Le questionnaire DN4 a été utilisé pour le diagnostic de la DN chez les 200 patients souffrant de lombalgie ou de LC inclus dans cette étude. Résultats: Des 200 patients (147 femmes et 53 hommes) inclus dans l'étude, la DN était présente chez 92 (46\%). L'âge moyen des 92 patients (67 femmes vs 25 hommes; $p=0.04$ ) était de 55,5 \pm 12.4 ans (femmes 55,2 $\pm 12,8$ vs. Hommes 54,6 $\pm 11,4 ; \mathrm{p}=0.5$ ). Les items de la DN retrouvés étaient essentiellement : brûlures $(n=67 ; 72,8 \%)$; décharges électriques $(n=64 ; 69,6 \%)$; fourmillements $(n=90 ; 97,8 \%)$; picotements $(n=57 ; 62 \%)$; engourdissement $(n=89 ; 96,7 \%)$; hypoesthésie $(\mathrm{n}=52 ; 56,5 \%)$. Les facteurs significativement associés à l'existence de DN au cours des LC étaient dominés par l'âge $(\mathrm{p}=0,005)$, la durée d'évolution de la lombalgie $(p=0,04)$, l'HTA $(p=0,001)$, les radiculalgies $(p=0,00002)$ et l'antécédent de lomboradiculalgie (0,000000).

Conclusion : La DN est fréquente chez des patients souffrant de LC à Lomé. L'âge, la durée d'évolution de la lombalgie, l'antécédent de lomboradiculalgie, la prise antérieure d'AINS, l'IMC, l'intensité de la douleur et les radiculalgies semblent prédictives de la survenue de ces DN.

Mots clés: lombalgie, douleur neuropathique, Afrique subsaharienne 


\section{Introduction}

La lombalgie commune constitue un véritable problème de santé publique. Elle est le premier motif de consultation en rhumatologie (Houzou $\mathrm{P}$ et al, 2013). Diverses études témoignent qu'elle constitue également un problème majeur en milieu de travail à cause des conséquences socioprofessionnelles importantes et du coût élevé induit pour la société par l'absentéisme et par la consommation médicale qu'elle entraine (Guo HR et al, 1995 ; Kazuhiro $\mathrm{K}$ et al, 2001 ; Vignier C et al, 1987). Les douleurs neuropathiques sont fréquentes en rhumatologie, essentiellement dans les sciatiques chroniques, souvent intriquées avec les douleurs par excès de nociception. Dans certains cas, elles peuvent expliquer les difficultés d'expression de la douleur par les patients et l'inefficacité des traitements antalgiques habituels (Perrot $S$ et al, 2002). La douleur neuropathique se définit comme la conséquence directe d'une lésion ou d'une maladie touchant le système somato-sensoriel (Treede RD et al, 2008). Sa Prévalence dans la population générale varie de 6 à $8 \%$ (Torrance $\mathrm{N}$ et al, 2006 ; Bouhassira D et al, 2008). Des études épidémiologiques dans les pays occidentaux ont montré que 20 à $35 \%$ des patients souffrant de lombalgies ont une DN (Freynhagen R et al, 2006 ; Freynhagen R et al, 2009). La prévalence de la $\mathrm{DN}$ au cours de la lomboradiculalgie est peu étudiée en Afrique (Ouedraogo DD et al, 2012 ; Nasreddine A et al, 2012 ; Doualla BM et al, 2013). Aucune étude sur ce sujet n'a été réalisée au Togo d'où l'intérêt de cette étude qui a pour objectif de déterminer la prévalence et les facteurs associés à la douleur neuropathique (DN) chez des patients ayant une lomboradiculalgie commune (LC) dans un pays en développement.

\section{Patients et methodes}

Il s'est agi d'une étude transversale qui s'est déroulée de mai à juillet 2016 (3 mois) dans les services de rhumatologie, de neurologie et de neurochirurgie du CHU Sylvanus Olympio de Lomé et dans le service de rhumatologie du CHR Lomé-Commune (Togo). Tous les patients ayant consulté pour lombalgie ou lomboradiculalgie commune évoluant depuis plus de 3 mois ont été inclus de manière consécutive. Les patients avec lombalgies chroniques d'origine inflammatoire, infectieuse ou néoplasique ont été exclus de cette étude. Les patients diabétiques ont également été exclus.Pour chaque patient, les données ont été recueillies à l'aide d'un questionnaire pré-établi comportant : les informations sociodémographiques (âge, sexe, profession, niveau d'instruction, région d'origine, langue parlée) ; les antécédents pathologiques (hypertension artérielle, diabète, affection neurologique préexistante, chirurgie lombaire) ; les items du questionnaire DN4 ; les éléments contributifs de l'examen clinique (siège de la douleur lombaire, 
présence ou non d'une irradiation dans le membre inferieur dépassant le genou, son trajet radiculaire, les signes déficitaires, conflit disco radiculaire, poids en kilogrammes, taille en centimètres) ; les données de l'imagerie réalisée (radiographies du rachis lombaire de face et de profil en charge, tomodensitométrie lombaire); la biologie (vitesse de sédimentation en millimètres) ; les traitements en cours avant l'inclusion dans l'étude et les traitements prescrits. La lombalgie était considérée comme chronique après une durée de 3 mois ou plus d'évolution. La douleur neuropathique était définie par un score $\geq 4$ au questionnaire DN4 (sensibilité de 82,9\% et spécificité de 89,9\%) (Bouhassira D et al, 2005). L'intensité de la douleur nociceptive a été mesurée grâce à une échelle numérique(EN) : $0=$ pas de douleur ; $10=$ douleur maximale imaginable. La douleur était classée comme faible (EN, 1-3), modérée (EN, 4-6) ou forte (EN, 7-10). La Classification de l'indice de masse corporelle (IMC) a été effectuée conformément aux critères de l'OMS. Les données ont été analysées avec le logiciel Epi Info 3.5.1. Les résultats ont été exprimés en termes de fréquence et de moyennes \pm écart-type. Les tests d'Anova et de Fischer ont été utilisés. Le $\mathrm{p}<0.05$ a été considéré comme significatif.

\section{Resultats}

\section{Caractéristiques de la population étudiée}

Deux cent sept patients ont été recueillis au cours de la période d'étude, dont 140 (70\%) de la consultation de Rhumatologie, 45 (22,5\%) de la consultation de Neurochirurgie et 15 (7,5\%) de la consultation de Neurologie. Selon le niveau d'instruction, on comptait 53 analphabètes $(26,5 \%)$ et 147 instruits $(30 \%$ du niveau primaire, $33 \%$ du niveau secondaire et $10,5 \%$ du niveau universitaire). Le questionnaire a été administré en mina chez 11 patients $(57,5 \%)$, en français chez 84 patients $(42 \%)$ et en anglais chez un patient $(0,5 \%)$.Cinquante-trois patients $(26,5 \%)$ étaient des hommes et $147(73,5 \%$ ) des femmes donnant une sex-ratio de 0,36 . L'âge moyen de nos patients était de $53 \pm 13$ ans avec des extrêmes de 24 et 86 ans. La tranche d'âge de 50 à 59 ans était la plus représentée dans $32 \%$ des cas. Les professions les plus fréquentes étaient celles des revendeuses et des ménagères respectivement dans $24,5 \%$ des cas et $15 \%$ des cas. La durée moyenne d'évolution de la lombalgie était de 55,7 \pm 78,7 mois avec des extrêmes de 3 mois et 492 mois. La lombalgie chronique évoluait depuis 3 mois à 1 an dans $23,5 \%$ des cas, entre 1 et 5 ans dans 48,5\% des cas et depuis plus de 5 ans dans $28 \%$ des cas. L'installation de la lombalgie était progressive chez 175 patients $(87,5 \%)$. L'intensité moyenne de la douleur était de $7 \pm 1,9$ avec extrêmes de 1 et 10 sur 10 . Cent quatre-vingt- dix- huit patients $(99 \%)$ avaient de la douleur modérée à sévère. 
Une irradiation radiculaire était notée chez 177 patients $(88,5 \%)$ de type L4 dans $20 \%$ des cas, L5 dans $48,5 \%$ des cas, S1 dans $9 \%$ des cas, et tronquée dans $11 \%$ des cas. Le signe de Lasègue était positif chez 70 patients (45,7\%). L'indice de Schöber a été mesuré chez 190 patients ; cent trente patients $(68,4 \%)$ avaient un indice Schöber qui était supérieur ou égal à 4 . Les antécédents contributifs étaient à type de lomboradiculalgie chez 120 patients $(60 \%)$, de chirurgie lombaire chez neuf patients $(4,5 \%)$, de traumatisme rachidien chez trois patients $(1,5 \%)$. Soixante- huit patients (34\%) n'avaient aucun antécédent de lombalgie et 51 patients $(25,5 \%)$ étaient hypertendus connus. L'indice de masse corporelle moyen était de $27,6 \pm 5,6 \mathrm{~kg} / \mathrm{m}^{2}$ avec des extrêmes de 17,9 et $48 \mathrm{~kg} / \mathrm{m}^{2}$. Nous avons noté un IMC normal chez 75 patients $(37,5 \%)$ un surpoids chez 64 patients $(32,5 \%)$ et une obésité chez 61 patients (30,5\%). Les étiologies des lomboradiculalgies communes chroniques objectivées par l'imagerie du rachis lombaire sont résumées dans le tableau 1.

Tableau 1 : Lésions radiologiques des lomboradiculalgies communes chroniques

\begin{tabular}{|c|c|c|}
\hline & $\begin{array}{c}\text { Nombre } \\
(\mathrm{N})\end{array}$ & $\begin{array}{c}\text { Pourcentage } \\
(\%)\end{array}$ \\
\hline $\begin{array}{l}\text { Radiographie } \\
\text {-Discarthrose } \\
\text {-Lombarthrose } \\
\text {-Spondylolisthésis } \\
\text {-Vide discal } \\
\text {-Aucune lésion }\end{array}$ & $\begin{array}{l}169 \\
75 \\
38 \\
04 \\
20 \\
\end{array}$ & $\begin{array}{l}84,5 \\
37,5 \\
19,0 \\
02,0 \\
10,0\end{array}$ \\
\hline $\begin{array}{l}\text { Tomodensitométrie ou Imagerie par résonance } \\
\text { magnétique } \\
\text {-Canal lombaire rétréci } \\
\text {-Arthrose inter apophysaire postérieure } \\
\text {-Hernie discale }\end{array}$ & $\begin{array}{l}13 \\
12 \\
10\end{array}$ & $\begin{array}{l}06,5 \\
06,0 \\
05,0\end{array}$ \\
\hline
\end{tabular}

Cent quatre-vingt patients avaient reçu un traitement avant la collecte. Parmi ceux-ci, 170 ont noté une légère amélioration puis une reprise de la douleur. Les dix autres n'avaient noté aucune amélioration. Les médicaments les plus utilisés étaient les antalgiques $(63,3 \%)$, les antiinflammatoires non stéroïdiens $(43,3 \%)$, les myorelaxants $(21,1 \%)$ et les vitamines $(27,7 \%)$. Les antidépresseurs (amitriptyline, clomipramine) et antiépileptiques (gabapentine, prégabaline) avaient été prescrit chez 40 patients $(22,2 \%)$.

\section{Caractéristiques des patients avec des douleurs neuropathiques}

Quatre-vingt-douze patients (46\%) souffrant de lomboradiculalgie commune chronique avaient une douleur neuropathique. Le score DN4 moyen était de 5,4 $\pm 1,4$. La prévalence des items du DN4 chez les patients ayant un score $\geq 4$ sont présentées dans le tableau 2 . 
Tableau 2 : Prévalence des items du DN4 chez les patients ayant une douleur neuropathique

\begin{tabular}{|l|c|c|}
\hline & Nombre $(\mathrm{N})$ & Pourcentage (\%) \\
\hline Brûlure & 67 & 72,8 \\
\hline Sensation de froid douloureux & 15 & 16,3 \\
\hline Décharges électriques & 64 & 69,6 \\
\hline Fourmillements & 90 & 97,8 \\
\hline Picotements & 57 & 62,0 \\
\hline Engourdissements & 89 & 96,7 \\
\hline Démangeaisons & 17 & 18,5 \\
\hline Hypoesthésie au tact & 52 & 56,5 \\
\hline Hypoesthésie à la piqûre & 50 & 54,3 \\
\hline Frottement & 14 & 15,2 \\
\hline
\end{tabular}

Les 92 patients se répartissaient en 67 femmes $(72,8 \%)$ et 25 hommes $(27,2 \%)$ sans différence significative ( $p>0,05)$. Leur âge moyen était de 55,5 $\pm 12,4$ ans avec des extrêmes de 24 et 86 ans. L'âge moyen des hommes était de 54,6 $\pm 11,4$ ans avec des extrêmes de 30 et 71 ans et celui des femmes de $55,2 \pm 12,8$ ans avec des extrêmes de 24 et 86 ans. La tranche d'âge de 50 à 59 ans était la plus représentée dans $29,3 \%$. La durée moyenne d'évolution était de 59,4 $\pm 70,5$ mois avec des extrêmes de 3 mois à 4lans. L'intensité moyenne de la douleur était de $7 \pm 1,6$ avec extrêmes de 3 et 8 sur 10 . La douleur était forte dans $83,7 \%$ des cas et modérée dans $13 \%$ des cas. L'indice de masse corporelle moyen était de $27,1 \pm 5,3 \mathrm{~kg} / \mathrm{m}^{2}$ avec des extrêmes de 17,9 et $48 \mathrm{~kg} / \mathrm{m}^{2}$. Nous avons noté un surpoids chez 38 patients $(41,3 \%)$ et une obésité chez 22 patients $(23,9 \%)$. La lomboradiculalgie était notée chez 91 patients $(98,9 \%)$ avec DN. Le trajet de la douleur était de type L4 dans $21,7 \%$ des cas, L5 dans 47,8\% des cas, S1 dans $12 \%$ des cas et tronqué dans $17,4 \%$ des cas. A l'inclusion, les 92 patients avec DN avaient reçu avaient reçu des antalgiques $(45 ; 49 \%)$, des AINS $(18 ; 19,5 \%)$, des infiltrations de corticoïdes $(59 ; 64 \%)$, des myorelaxants $(22 ; 24 \%)$ et des antidépresseurs tricycliques $(20 ; 21,7 \%)$ et antiépileptiques $(10 ; 10,8 \%)$. Les facteurs de risques qui favorisaient de façon significative l'apparition de de douleur neuropathique étaient : l'âge $(\mathrm{p}=0,005)$, la durée d'évolution de la lombalgie $(\mathrm{p}=0,04)$, l'antécédent de lomboradiculalgie $(\mathrm{p}=0,0000)$, l'HTA $(\mathrm{p}=0,001)$, l'intensité de la douleur $(\mathrm{p}=0,02)$, l'IMC $(\mathrm{p}=0,04)$, la prise antérieure d'AINS $(\mathrm{p}=0,0000002)$, et l'existence d'une irradiation $(\mathrm{p}=0,0002)$. Ces résultats sont explicités dans les tableaux 3 à 5 .

Tableau 3 : Association significative entre la douleur neuropathique et l'âge, l'intensité de la douleur et la durée d'évolution de la lombalgie

\begin{tabular}{|l|c|c|c|}
\hline & $\begin{array}{c}\text { DN4 } \leq 3 \\
\mathrm{~m} \pm \mathrm{ET}^{*}\end{array}$ & $\begin{array}{c}\mathrm{DN} 44 \\
\mathrm{~m} \pm \mathrm{ET}\end{array}$ & $\begin{array}{c}\text { Probabilité } \\
(\mathrm{p})\end{array}$ \\
\hline Age (ans) & $49,8 \pm 13$ & $55,5 \pm 12,4$ & 0,005 \\
\hline Intensité & $07,0 \pm 1,9$ & $07,0 \pm 1,6$ & 0,02 \\
\hline Durée d'évolution de la lombalgie & & & \\
\hline
\end{tabular}




\begin{tabular}{|l|c|c|c|}
\hline (mois) & $55,7 \pm 78,7$ & $59,4 \pm 70,5$ & 0,04 \\
\hline \multicolumn{4}{|c|}{$*$ moyenne \pm Ecart type }
\end{tabular}

Tableau 4 : Association entre la douleur neuropathique et les antécédents

\begin{tabular}{|l|c|c|c|}
\hline & DN4 $\leq 3$ & DN4 $\geq 4$ & $\begin{array}{c}\text { Probabilité } \\
(\mathrm{p})\end{array}$ \\
\hline ATCD de lomboradiculalgie & & & 0,00000000 \\
-Oui & 28 & 92 & \\
-Non & 80 & 0 & 0,5 \\
\hline Chirurgie lombaire & 04 & 05 & \\
-Oui & 104 & 87 & 0,4 \\
-Non & 01 & 02 & \\
\hline Traumatisme lombaire & 107 & 90 & 0,001 \\
-Oui & 18 & 33 & \\
-Non & 90 & 59 & \\
\hline HTA & &
\end{tabular}

Tableau 5 : Association entre la douleur neuropathique et les caractéristiques clinique et thérapeutique

\begin{tabular}{|l|c|c|c|}
\hline & DN4 $\leq 3$ & DN4 $\geq 4$ & $\begin{array}{c}\text { Probabilité } \\
(\mathrm{p})\end{array}$ \\
\hline Mode de debut & 15 & 10 & 0,2 \\
-Brutal & 93 & 82 & \\
-Progressif & 86 & 91 & 0,00002 \\
\hline Irradiation & 22 & 01 & \\
-Oui & 43 & 32 & 0,04 \\
-Non & 26 & 38 & \\
\hline IMC & 39 & 22 & 0,03 \\
-18-24,9 & 69 & 45 & \\
- 25-29,9 & 39 & 47 & 0,0000002 \\
- 30 & 60 & 18 & \\
\hline Prise d'Antalgiques & 48 & 74 & \\
-Oui & & & \\
-Non & & & \\
\hline Prise d'AINS & & & \\
-Oui & & & \\
-Non & &
\end{tabular}

\section{Discussion}

La prévalence de la DN chez les patients souffrant de LC à l'aide du questionnaire DN4 était de $46 \%$ et les principaux facteurs significativement associés à l'existence de cette DN au cours des LC étaient l'âge, la durée d'évolution de la lombalgie, les radiculalgies et l'antécédent de lomboradiculalgie.

Cette prévalence est voisine de celle retrouvée par Ouedraogo et al au Burkina-Faso (Ouedraogo DD et al, 2012) et de Nasreddine et al au Maroc 
(Nasreddine A et al, 2012) qui ont utilisé également le DN4. Nos résultats se rapprochent également de ceux trouvés au Proche Orient par Hassan et al (Hassan AE et al, 2004) et El Sissi et al (El Sissi W et al, 2010) qui eux ont utilisés le Leeds assessment of neuropathic symptoms and signs (LANSS)(Bennett $M$ et al, 2001). Nos résultats sont supérieurs à ceux obtenus dans les pays occidentaux (Freynhagen R et al, 2006 ; Freynhagen R et al, 2009) et au Cameroun (Doualla BM et al, 2013) mais inférieurs à celui de Ridene et al en Tunisie (Ridene $\mathrm{M}$ et al, 2009). Cette forte prévalence des DN dans les lombalgies chroniques pourrait s'expliquer par la forte prévalence des lombalgies chroniques dans la population générale.Le questionnaire DN4 a motivé notre choix car c'est un questionnaire simple d'utilisation et adapté au profil socioprofessionnel de nos patients.

Les principales caractéristiques de la DN dans notre étude étaient les fourmillements, les engourdissements, les brûlures et les décharges électriques. Ce résultat est similaire aux données de la littérature (Ouedraogo DD et al, 2012; Nasreddine A et al, 2012; Doualla BM et al, 2013). Plusieurs facteurs de risque interviennent dans la survenue de DN chez les patients souffrant de LC. Dans notre étude, l'âge moyen de nos lombalgiques avec DN était de 55,5 ans et était significativement associé à la survenue de DN au cours des LC. Ce résultat est similaire à ceux d'Hassan et al (Hassan AE et al, 2004) et de Kaki et al (Kaki AM et al, 2005).Ouedraogo et al (Ouedraogo DD et al, 2012)et Doualla et al(Doualla BM et al, 2013)ont rapporté respectivement un âge moyen de 47,58 ans et 57,7 ans mais l'âge n'était pas significativement associé.Dans notre étude et dans celles d'autres auteurs (Ouedraogo DD et al, 2012 ;Doualla BM et al, 2013 ;Hassan AE et al, 2004 ; Ridene $\mathrm{M}$ et al, 2009) malgré la prédominance féminine, le sexe n'était pas significativement associé à l'existence de DN au cours des LC contrairement à celles de El sissi et al et de Kaki et al. (El Sissi W et al, 2010; Kaki AM et al, 2005).La durée d'évolution de la LC était significativement associé contrairement à d'autres études africaines (Ouedraogo DD et al, 2012 ; Doualla BM et al, 2013). La plupart des études (Ouedraogo DD et al, 2012 ; Doualla BM et al, 2013 ; Attal N et al, 2006), confirme que la présence de radiculalgie est un facteur associé à la survenue de DN au cours des LC. Dans notre étude les autres facteurs significativement associés à l'existence de DN au cours des LC étaient l'antécédent de lomboradiculalgie, l'IMC, l'intensité de la douleur, l'hypertension antérieure et la prise antérieure de médicaments. Ces résultats sont similaires à ceux décrits dans d'autres études comme pour l'IMC (Kaki AM et al, 2005), l'HTA (El Sissi W et al, 2004; Kaki AM et al, 2005) et la prise antérieure de médicaments (Kaki AM et al, 2005). L'antécédent de chirurgie lombaire n'était pas significativement associé dans notre étude 
contrairement aux études d'Hassan et al et Kaki et al (Hassan AE et al, 2004); Kaki AM et al, 2005).

Les antidépresseurs et antiépileptiques ont été utilisés chez 30 $(32,6 \%)$ des 92 patients ayant une DN. Ce résultat est similaire à celui de Doualla et al (Doualla BM et al, 2013) mais supérieur à celui d'Ouedraogo et al (Ouedraogo DD et al, 2012 ); ceci témoigne d'une part du non utilisation en pratique courante du questionnaire DN4 chez les patients souffrant de LC par le nombre restreint de rhumatologues (08), de neurologues (09) et de neurochirurgiens (05) au Togo, et d'autre part par la faible accessibilité financière et d'approvisionnement à la gabapentine et à la prégabaline. Les antidépresseurs et antiépileptiques constituent le traitement de $1^{\text {ère }}$ ligne selon les recommandations européennes (Attal N et al, 2006).

\section{Conclusion}

La DN est fréquente chez des patients de la cinquantaine, de sexe féminin souffrant de LC à Lomé. L'âge, la durée d'évolution de la lombalgie, l'antécédent de lomboradiculalgie, la prise antérieure d'AINS, l'IMC, l'intensité de la douleur et les radiculalgies semblent prédictives de la survenue de ces DN. Les moyens diagnostiques des DN devraient être enseignés et connus de tous les praticiens afin de permettre un diagnostic précoce et une prise en charge adéquate par les antidépresseurs et des antiépileptiques.

\section{References:}

1. Houzou P, Oniankitan O, Kakpovi K, Koffi-Tessio V, Tagbor KC, Fianyo E, Mijiyawa M. Profil des affections rhumatismales chez 13517 patients ouest africains (2013). Tunis Méd ;91: 16-20.

2. Guo HR, Tanaka S, Cameron LL, Seligman PJ, Behrens VJ, Ger J, et al. Back pain among workers in the United States: national estimates and workers at high risk (1995). Am J Ind Med; 28:591-602

3. Kazuhiro K, Yasumasa S, Masabumi M. An epidemiological study on occupational low back pain among people who work in construction (2001). J Nippon Med Sch; 68: 310-17.

4. Vignier C, Brenier E, Carie S. Conséquences socioprofessionnelles des lombalgies en milieu de travail (1987). Document pour le médecin du travail; 29 :17-24.

5. Perrot S, Richard Trèves R. Les douleurs neuropathiques en rhumatologie (2002). Rev Rhum [Ed Fr]; 69 : 961-70.

6. Treede RD, Jensen TS, Campbell JN, Cruccu G, Dostrovsky JO, Griffin JW, et al. Neuropathic pain: redefinition and a grading system for clinical and researchpurposes (2008). Neurology; 70:1630-5. 
7. Torrance N, Smith BH, Bennett MI, Lee AJ. The epidemiology of chronic pain of predominantlyneuropathicorigin. Results from a general population survey (2006). J Pain;7:281-9.

8. Bouhassira D, Lanteri-Minet M, Attal N, Laurent B, Touboul C. Prevalence of chronic pain withneuropathiccharacteristics in the general population (2008). Pain;136:380-7.

9. Freynhagen R, Baron R, Tölle T, Stemmler E, Gockel U, Stevens $\mathrm{M}$, andMaier C. Screening of neuropathic pain components in patients withchronic back pain associatedwith nerve root compression: a prospective observational pilot study (IMPORT) (2006). Curr Med ResOpin; 22:529-37.

10. Freynhagen R, Baron R. The evaluation of neuropathic pain components low back pain (2009). Curr Pain HeadacheRep 2009;13:185-90.

11. Ouédraogo DD, Nonguierma V, Napon C, Kabré A, Tiéno H, Guira O, Kaboré J, Drabo JY. Prevalence of neuropathic pain among Black African patients suffering from commonlow back pain (2012). Rheumatol Int.; 32(7):2149-53.

12. Nasreddine A, Rachidi W, jouani S, Mkinsi O. La douleur neuropathique en consultation de rhumatologie (2012). Rev Rhum;79 (Abst. Lu 36) : A146.

13. Doualla BM, LumaNamme H, TchaleuNguenkam B, Kwedi F, KemtaLekpa F, Memopi M, NgandeuSingwe M. Prevalence of neuropathic pain among patients withchroniclow back pain in Douala, Cameroon (2013). Rev Mar Rhum; 26: 26-30.

14. Bouhassira D, Attal N, Alchaar H, Boureau F, Brochet B, Bruxelle J, et al. Comparison of pain syndromes associatedwithnervous or somaticlesions and development of a new neuropathic pain diagnostic questionnaire (DN4) (2005). Pain; 114:29-36.

15. Hassan AE, Hosny A S, Yehia M B, Khalid I A, Marwan W N, Manzor S. K et al. Prevalence of neuropathic pain among patients suffering from chronic low back pain in Saudi Arabia (2004). Saudi Med J; 25: 1986-90.

16. El Sissi W, Amaout A, Chaarani MW, Fouad M, El Assuity W, Zalzala M, Dershaby YE, Youseif E. Prevalence of neuropathic pain among patients withchroniclow-back pain in the Arabian Gulf Regionassessedusing the Leeds Assessment of Neuropathic

17. Symptoms and Signs pain scale (2010). J Int Med Res; 38:2135-45.

18. Bennett M. The LANSS Pain Scale: the Leeds assessment of neuropathicsymptoms and signs (2001). Pain; 92:147-157.

19. Ridene M, Tekaya R, Ben hadj Yahia C et al. Neuropathic pain in commonradiculalgy (2009). Rev Rhum [abstract]; 76:986. 
20. Kaki AM, El-Yaski ZA, Youseif E. Identifyingneuropathic pain among patients withchroniclow-back pain: use of the Leeds Assessment of NeuropathicSymptoms and Signs pain scale (2005). Reg Anesth Pain Med; 30:422-428.

21. Attal N, Haanpaa M, Hanson $P$ et al. EFNS guidelines on pharmacologicaltreatment of neuropathic pain (2006). Eur J Neurology; 13:1153-1169. 